Electrical and dielectric properties of carbon black filled co-continuous two-phase polymer blends

This article has been downloaded from IOPscience. Please scroll down to see the full text article.

1999 J. Phys. D: Appl. Phys. 321517

(http://iopscience.iop.org/0022-3727/32/13/313)

View the table of contents for this issue, or go to the journal homepage for more

Download details:

IP Address: 139.165.109.87

The article was downloaded on 10/12/2012 at 10:46

Please note that terms and conditions apply. 


\title{
Electrical and dielectric properties of carbon black filled co-continuous two-phase polymer blends
}

\author{
C Calberg $\dagger$, S Blacher $\ddagger, F$ Gubbels $\dagger$, F Brouers $\ddagger$, R Deltour $§$ and \\ $\mathbf{R}$ Jérôme $\dagger$
}

$†$ Center for Education and Research on Macromolecules (CERM), University of Liège, Sart-Tilman, B6, 4000 Liège, Belgium

\$ Service de Génie Chimique et Physique des Matériaux, University of Liège, Sart-Tilman, B6, 4000 Liège, Belgium

$\S$ Service de Physique des Solides, Université Libre de Bruxelles, CP 233, Blvd du Triomphe, 1050 Bruxelles, Belgium

Received 21 September 1998, in final form 1 March 1999

\begin{abstract}
The electrical and dielectric properties of co-continuous polystyrene (PS)/poly(methylmethacrylate)(PMMA) blends loaded with carbon black (CB) of a special grade (BP 1000) have been studied. They strongly depend on the selective localization of the $\mathrm{CB}$ particles at the blend interface quite consistently with the double percolation concept. The interfacial localization of CB contributes to the stabilization of the phase morphology against thermal post treatment. Nevertheless, the sample annealing has a very favourable effect on the percolation threshold that decreases. The composition range in which phases are co-continuous is also increased by the addition of the filler. Dispersion of the conductance and the dielectric constant has been measured in the wide frequency range $10^{-4}-10^{8} \mathrm{~Hz}$ for blends containing $0-5 \mathrm{wt} \% \mathrm{CB}$. On the basis of these results, the mechanism of electrical transport (trapping, hopping, tunnelling, percolation) has been discussed in relation to the $\mathrm{CB}$ loading and the optimum loading has been identified for the electrical and dielectric properties to be stable and reproducible.
\end{abstract}

\section{Introduction}

Imparting electrical conductivity to otherwise insulating polymers is nowadays a very valuable goal. For instance, dispersion of conducting carbon black (CB) particles in synthetic polymers is a very efficient way of providing them with semiconductivity and antistatic properties [1-6] which are required by important technological developments, such as electrical wires and pipelines. From technical and economical viewpoints, it is desirable to decrease the $\mathrm{CB}$ percolation threshold $\left(p_{c}\right)$ as much as possible, i.e. the lowest concentration required for the conducting particles to form continuous conducting paths. Indeed, too large an amount of $\mathrm{CB}$ has a detrimental effect on the processing and mechanical properties of the final material, as well as on the cost/performance balance.

The insulator-conductor transition in polymer-CB composites is known to depend on the aggregation [7-9], structure, porosity, average size and size distribution of the conducting particles $[10,11]$, on polymer rheology $[8,9]$ and processing conditions. It has been previously shown that the selective localization of conducting particles in one of the phases or at best at the interface of a co-continuous two-phase polyblend is a very efficient strategy to decrease the CB percolation threshold [12-15]. It is worth pointing out that double percolation is at the origin of this situation: (i) percolation of the polymer phases and thus of their interface and (ii) percolation of the conducting particles in one phase or at the interface. The beneficial effect of the annealing of these ternary systems on the $\mathrm{CB}$ percolation threshold has also been reported when $\mathrm{CB}$ is localized in one phase.

Three major aims are envisaged in this paper. Since most of the previous studies have been conducted with polyethylene/polystyrene (PE/PS) blends [13-15], it is a valuable goal to extend the dual percolation concept to other binary blends, particularly to blends that contain a more polar polymer (PMMA) than PE. Since the electrical transport mechanism in multiphase materials is not very well known, it will be studied on the basis of the experimental data of dc conductivity, ac conductivity and the dielectric constant, in a wide range of frequencies, in close relationship with the polyblend morphology. Finally, the optimum CB loading has been searched for, which is thus the minimum loading able to impart the best electrical and dielectric stability and reproducibility. 


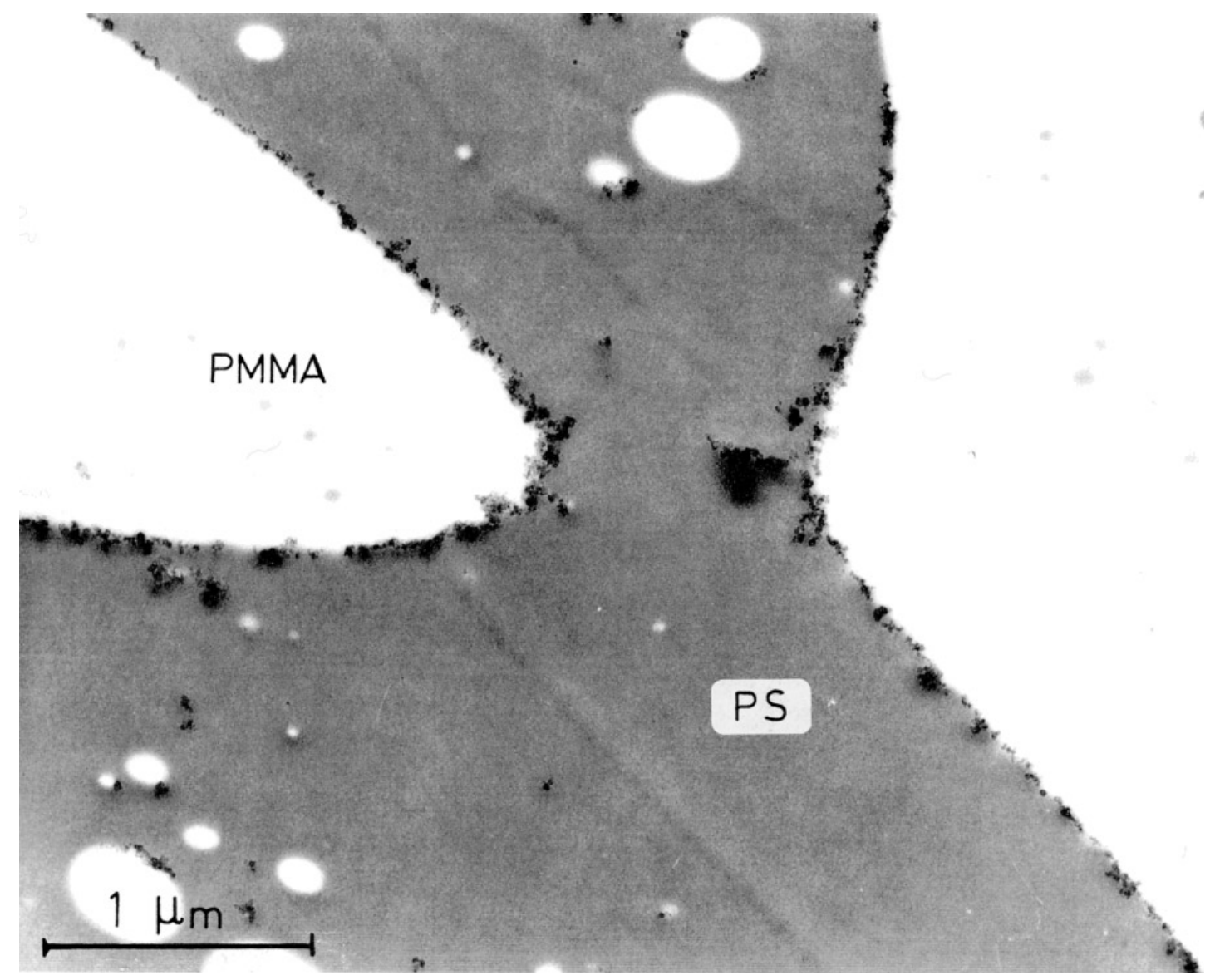

Figure 1. TEM micrograph of a 50/50 PS/PPMA blend filled with 2 wt $\%$ BP1000. The white nodules are PMMA sub-inclusions in the PS phase.

\section{Experimental details}

Mixing technique. Poly(methylmethacrylate) (PMMA: ICI Diakon MG102) and polystyrene (PS: BASF 158K) were melt blended in an internal mixer (Brabender Plasticorder) at $200{ }^{\circ} \mathrm{C}$ for $1 \mathrm{~min}$. CB (BP 1000: CABOT) was then added and the mixing rate was increased 1 min later, from $25 \mathrm{rpm}$ to $64 \mathrm{rpm}$ and maintained at $64 \mathrm{rpm}$ for $10 \mathrm{~min}$. $0.2 \mathrm{~cm}$ thick plates were compression moulded between aluminium foils at $260{ }^{\circ} \mathrm{C}$ for $10 \mathrm{~min}$, and cooled down to $25^{\circ} \mathrm{C}$ under pressure.

Extraction method. 2 or $3 \mathrm{~g}(\mathrm{w})$ of composite were immersed in glacial acetic acid at $60^{\circ} \mathrm{C}$ in order to dissolve PMMA selectively. The solvent was renewed $24 \mathrm{~h}$ later and the extraction repeated until PMMA no longer passed in solution. The sample was then dried under vacuum at $40^{\circ} \mathrm{C}$ until constant weight (w).

Phase morphology. The three-dimensional continuity of PS and PMMA phases in the parent polyblends was analysed by the selective extraction of the PMMA phase with acetic acid at $50^{\circ} \mathrm{C}$. When the extraction leaves the sample compact (no fragmentation into smaller pieces), PS is considered $100 \%$ continuous, whereas continuity of PMMA is quantified as the weight fraction of PMMA which is extracted. Conversely, when the sample falls apart, PMMA is $100 \%$ continuous and the PS continuity is estimated as the weight fraction of the biggest compact piece of PS released by the extraction process. Although contaminated by small PS fragments, this biggest piece is thought to be representative of the finite clusters defined by percolation theory.

Electrical measurements. The direct current (dc) electrical conductivity of filled polyblends was measured by the four-probe technique, so that it was free from the resistance of the sample/electrode contacts. Alternating current (ac) electrical conductivity was measured using two complementary devices: (a) the impedance analyser HP 4194A, supplied with the HP 41941B probe which allowed the $10^{3}-10^{8} \mathrm{~Hz}$ frequency range to be explored and (b) the frequency analyser (Schlumberger) SI 1255, supplied with the Chelsea Dielectric Interface (Model CDI 4t) for probing the $10^{-3}-10^{6} \mathrm{~Hz}$ frequency range.

\section{Results and discussion}

\subsection{Morphological analysis}

BP 1000, a strongly oxidized CB ( $\mathrm{pH}=2.5)$, has been dispersed into melted PS/PMMA blends. TEM micrographs show that $\mathrm{CB}$ is spontaneously localized at the polyblend interface (figure 1), indicating a lack of interaction of $\mathrm{CB}$ with each polymer phase. Filler localization in a two-phase polyblend can be predicted qualitatively from the wetting coefficients, as illustrated by Sumita et al [9] in the case of the interfacial localization of $\mathrm{CB}$ in PE/PMMA blends. 


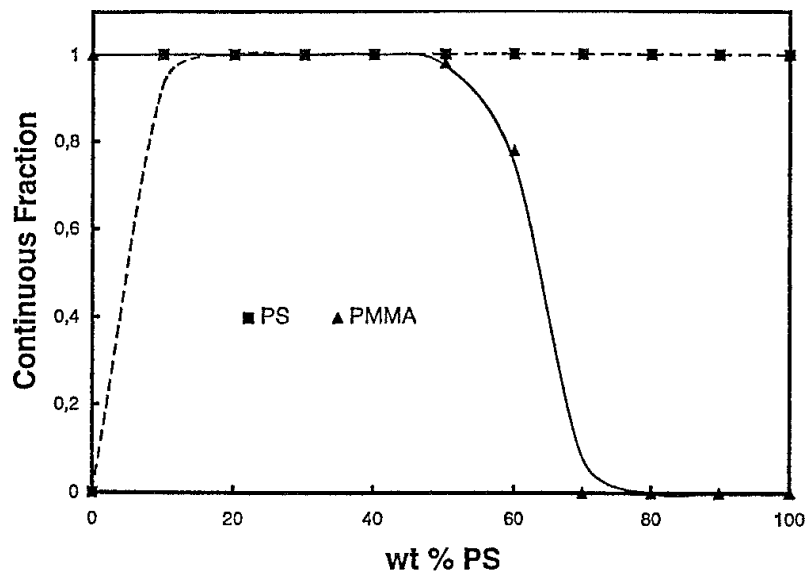

(a)

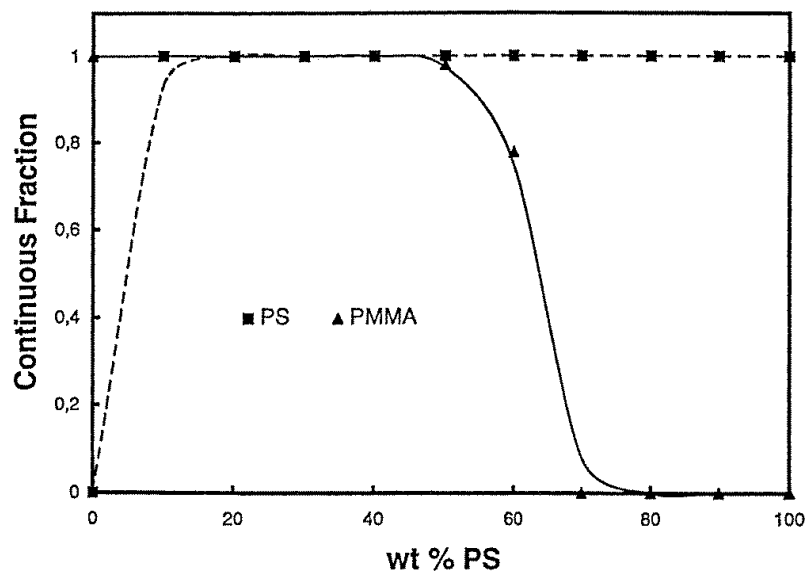

(c)

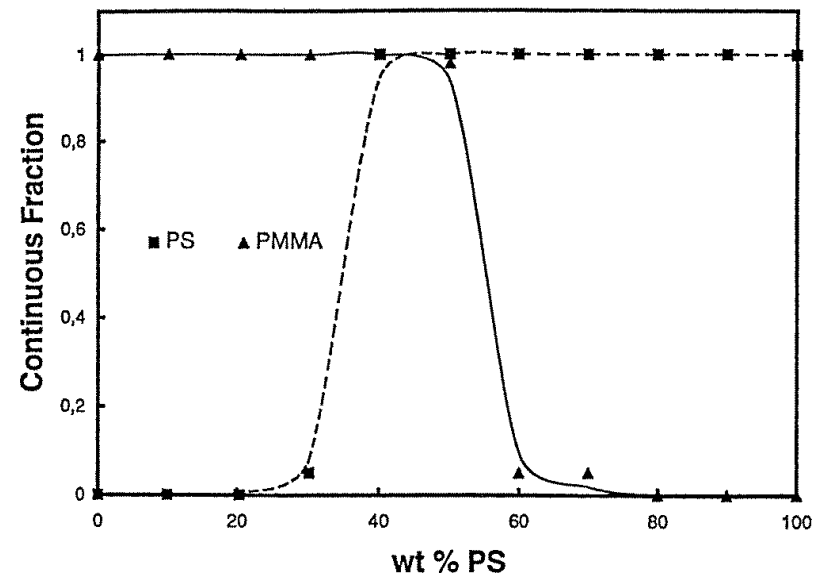

(b)

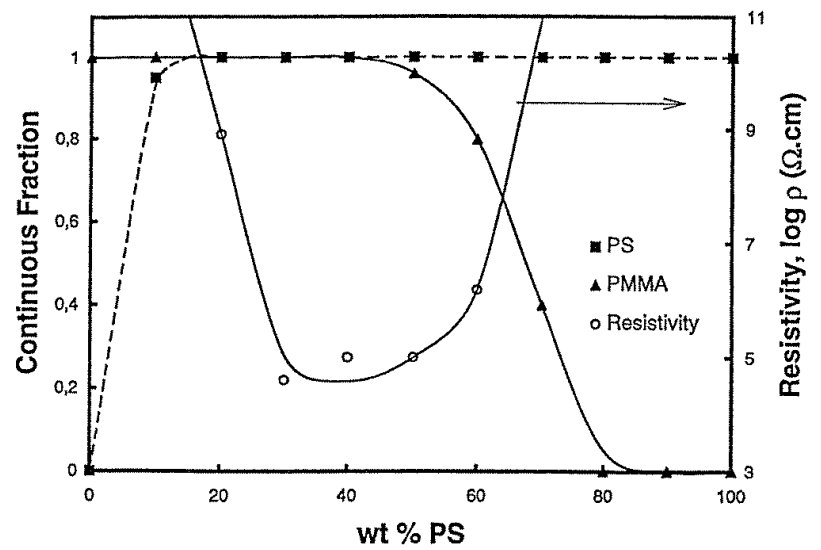

(d)

Figure 2. Dependence of the PS and PMMA phase continuity on the PS/PMMA blend composition: (a) no CB; (b) no CB but annealing for $10 \mathrm{~min}$ at $200{ }^{\circ} \mathrm{C}$; (c) $4 \mathrm{wt} \%$ BP 1000 ; (d) $4 \mathrm{wt} \%$ BP 1000 plus annealing for $10 \mathrm{~min}$ at $200^{\circ} \mathrm{C}$.

The specific localization at the interface of PS/PMMA blends is independent of the blend composition. Nevertheless, when the $\mathrm{CB}$ content exceeds about $4 \mathrm{wt} \% \mathrm{CB}$, the interface is saturated and part of the filler is found in the PMMA phase.

Since the electrical properties critically depend on the phase co-continuity and the interfacial area, it is worth studying the influence of $\mathrm{CB}$ content and blend annealing on the phase morphology. For this purpose, PS/PMMA blends of various compositions, filled or not with $\mathrm{CB}$, were prepared. Phase co-continuity has been studied by selective extraction of PMMA as explained in the experimental section. Ultramicrotomed sections have also been observed by TEM and the average size of the phases and the interfacial area have been calculated by image analysis as detailed elsewhere $[13,15]$.

Figures 2(a)-(d) compare the co-continuity domain for PS/PMMA samples, before and after loading with $4 \mathrm{wt} \%$ BP 1000, annealed or not. Annealing of the unloaded samples shifts the composition at the phase inversion from $30 / 70$ to $45 / 55$ PS/PMMA composition (figures 2(a) and (b)). When $4 \mathrm{wt} \% \mathrm{CB}$ is added, the domain of co-continuity is dramatically increased (cf figures 2(a) and (c)), which remains essentially stable against compression moulding at $200{ }^{\circ} \mathrm{C}$ for $10 \mathrm{~min}$ (cf figures 2(c) and (d)). Similar observations have been reported for PE/PS blends with
CB confined in one phase [13], and accounted for by the interfacial activity of $\mathrm{CB}$ and the increase in blend viscosity as result of the addition of $\mathrm{CB}$.

Figures 3(a) and (b) show how the mean size of the PS phase and the specific interfacial area for the 50/50 PS/PMMA blend depend on the compression moulding temperature, before and after loading with $4 \mathrm{wt} \% \mathrm{CB}$. Figure 3(a) illustrates the strong stabilization effect of $\mathrm{CB}$ that clearly slows down the coalescence of the phases. The effect of $\mathrm{CB}$ on the specific interfacial area depends on the moulding temperature. At $T<200{ }^{\circ} \mathrm{C}$, this area is higher in the absence of $\mathrm{CB}$, whereas the reverse situation is observed at $T>200^{\circ} \mathrm{C}$. This observation can be explained as follows. For the 50/50 PS/PMMA blend, the phase cocontinuity is incomplete since, in contrast to the PMMA phase which is continuous (100\%), the PS phase is only $80 \%$ continuous. The complete co-continuity is achieved when the polyblend is added to $4 \mathrm{wt} \% \mathrm{CB}$. Since the annealing at temperatures lower than $200^{\circ} \mathrm{C}$ does not change the phase morphology significantly, it is not surprising that the specific interfacial area is smaller for the completely co-continuous system (loaded blend) than for the same blend where PS partly forms dispersed phases in PMMA. At $T>200^{\circ} \mathrm{C}$, the phase coalescence occurs during annealing making the 50/50 PS/PMMA blends co-continuous (figure 2(b)) and decreasing 


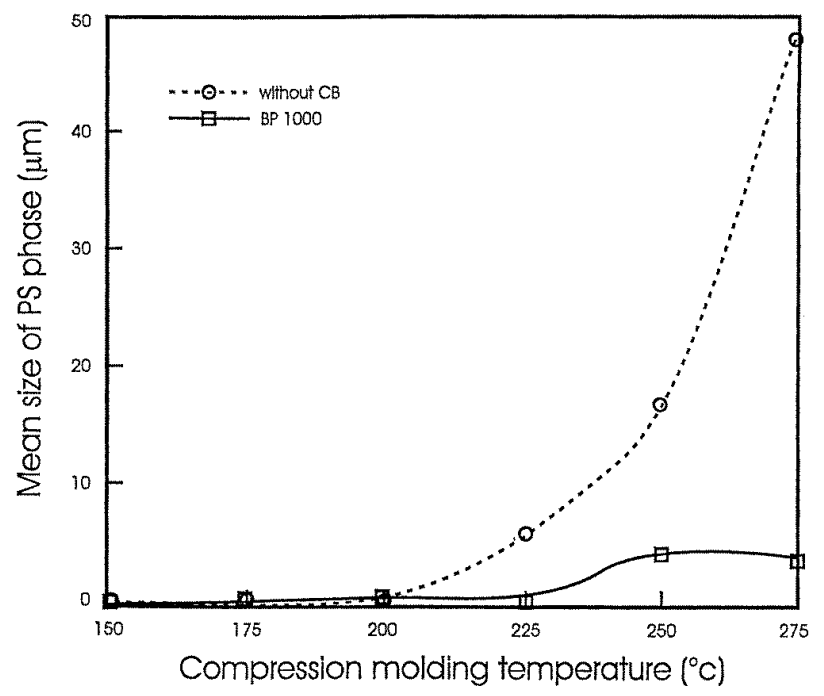

(a)

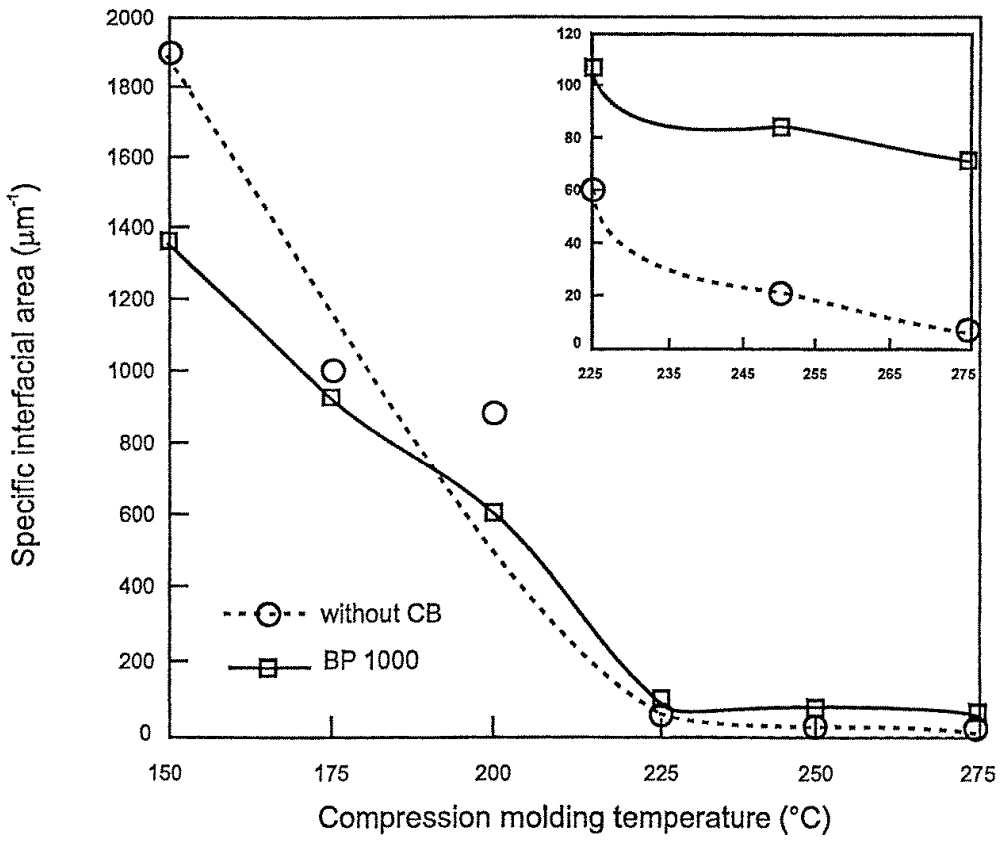

(b)

Figure 3. Effect of the compression moulding temperature on: (a) the mean size of the PS phase for the 50/50 PS/PMMA blend filled with 4 wt\% BP 1000; (b) the specific interfacial area for the same sample.

the specific interfacial area. The same effect is essentially limited for the loaded polyblend, whose the viscosity is increased by CB localized at the interface. Accordingly, the specific interfacial area becomes larger for the loaded polyblend compared to the unloaded counterpart.

\subsection{Electrical properties}

The electrical properties of polymer-CB composites are generally discussed on the basis of percolation theory [16-20]. In the close vicinity of the percolation threshold $\left(p_{c}\right)$ (the $\mathrm{CB}$ content $(p)$ at which the insulator-conductor transition occurs) the effective dc conductivity $\sigma_{e}$, and the dielectric constant $\varepsilon_{e}$, obey the following power laws close to $p_{c}[21,22]$

$$
\begin{gathered}
\sigma_{e}=\sigma_{m}\left|p-p_{c}\right|^{t} \quad \text { for } p>p_{c} \\
\varepsilon_{e}=\varepsilon_{d}\left|p-p_{c}\right|^{-s} \quad \text { for } p<p_{c} \text { and } p>p_{c}
\end{gathered}
$$

where $\sigma_{m}$ and $\varepsilon_{d}$ are the conductivity and the dielectric constant of the conductor and the insulator, respectively. The linear ac response has been discussed on the basis of the scaling form of the complex ac conductivity $[21,22]$

$$
\sum(p, \omega) \approx|\Delta p|^{t} g| \pm|\left(\frac{\mathrm{i} \omega}{\omega_{c}}\right)
$$

where $\mathrm{i}=\sqrt{-1}, \omega$ is the applied frequency, $g+$ and $g-$ are two different scaling functions for $p>p_{c}$ and $p<p_{c}$, 


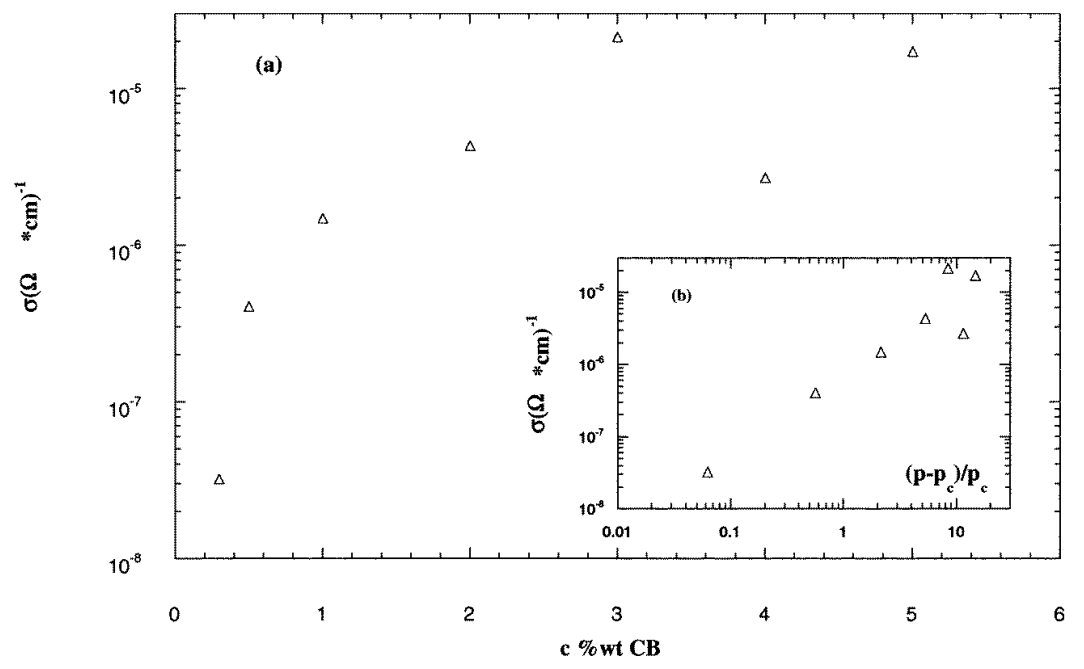

Figure 4. Plots of the dc conductivity for the 50/50 PS/PMMA blend against: (a) the filler content; (b) $\left|p-p_{c}\right| / p_{c}$.

respectively; $\Delta p=\left|p-p_{c}\right|$, and $\omega_{c}$ is a characteristic frequency scale expressed as follows

$$
\omega_{c}=\omega_{0}|\Delta p|^{s+t}
$$

with $\omega_{0}=\sigma_{m} / \varepsilon_{d}$. Based on the definition of $\sigma(\sigma=$ $\left.\operatorname{Re} \sum(p, \omega)\right)$ and $\varepsilon\left(\varepsilon=\operatorname{Im} \sum(p, \omega) / \omega\right)$, we have [22]

$$
\begin{aligned}
\sigma(p, \omega) \approx \omega^{x} & \text { for } p_{c} \approx p \\
\sigma(p, \omega) \approx \omega^{-y} & \text { for } p_{c} \approx p
\end{aligned}
$$

where $x=t /(s+t)$ and $x+y=1$. For two-dimensional (2D) systems $s=t \approx 1.3$. For three-dimensional (3D) systems $s \approx 0.7$ and $t \approx 2$.

It appears that the classical theory of percolation cannot account even qualitatively for most of the experimental data reported for thin granular films and conductor-insulator composites. The experimental $t, s$ and $x$ exponents may be quite different from the theoretical values, and conductivity often exhibits an exponential behaviour at $p<p_{c}$. The theory has been modified in order to account for the complex geometry and the structure of the aggregates [16]. It is also clear that a proper description of the electrical behaviour of CB-polymer composites cannot dismiss the tunnelling effect between the conducting aggregates [16,19,23-30]. Tunnelling and other transport mechanisms (trapping, hopping) that can occur at low loading will be considered in the discussion of the ac data.

3.2.1. de measurements. The electrical resistivity of PS/PMMA blends of various compositions and loaded with $4 \mathrm{wt} \%$ BP 1000 has been measured and compared to the (co) continuity of the phases in figure 2(d). The minimum resistivity fits pretty well with the blend composition at the phase inversion, i.e. the 50/50 composition. This observation confirms that the conductivity is critically driven by a double percolation phenomenon since when this condition is fulfilled, conductivity is higher than that measured for each polymer loaded with $4 \mathrm{wt} \% \mathrm{CB}$. The inverse dependence of resistivity on the extent of phase co-continuity observed in figure 2(d) also suggests that CB must be localized at the blend interface. Indeed, should the CB particles be localized in the PS phase, the resistivity would be smaller as soon as the PS is continuous (thus 20\% PS) and should increase sharply when the PS continuity is lost (beyond $40 \%$ PS), which does not match the data of figure 2 . The alternative argument that $\mathrm{CB}$ is in the PMMA phase does not hold either, since at least $7.5 \mathrm{wt} \% \mathrm{CB}$ would be required in order to make the 50/50 PS/PMMA blend conducting; the percolation threshold of BP 1000 in PMMA being $15 \mathrm{wt} \%$.

Figure 4(a) shows the dependence of the dc conductivity $\sigma_{e}$, on the $\mathrm{CB}$ content, for 50/50 PS/PMMA blends. Assuming that $p_{c}$ is in the $0.30-0.32 \mathrm{wt} \%$ range, equation (1) is fitted by the experimental data with the critical exponent $t=1.12 \pm 0.1$ (figure 4(b)). Assuming that $p_{c}$ is 0.25 , and discarding the first data point ( $p=0.30$ ), equation ( 1 ) is fitted with a critical exponent $t=1.3 \pm 0.1$. In the first case, the exponent is near to one which is predicted by the Bruggeman mean-field theory and is dimension independent [31]. In the second case, the exponent is near to 1.3 which is predicted by the classical percolation theory for $2 \mathrm{D}$ systems. The value of $t$ calculated in this work is thus in line with the interfacial localization of BP 1000 in the PS/PMMA blends.

3.2.2. ac measurements. In order to obtain a better insight into the electrical transport in the polymer-CB composites, it is rewarding to analyse the ac properties over a large range of frequencies. These ac measurements are expected to provide information about the percolation threshold, the dimensionality of the conducting paths and the conduction mechanism below and above the percolation threshold.

Figures 5(a) and (b) illustrate the frequency dependence $\left(10^{-4}-10^{8} \mathrm{~Hz}\right)$ of the conductance $\sigma$ and the dielectric constant of $\varepsilon$ the 50/50 PS/PMMA blends loaded with BP 1000 up to $5 \mathrm{wt} \%$ content. Two well-defined regimes emerge from these data, i.e. regime A $(0.5-5 \mathrm{wt} \% \mathrm{CB})$, well above the percolation threshold, and regime B (0-0.09 wt\% CB), well below this threshold.

It is not possible to assign a precise value to the percolation threshold, because the transition from regime $\mathrm{A}$ to regime $\mathrm{B}$ extends over a too broad a composition range, 


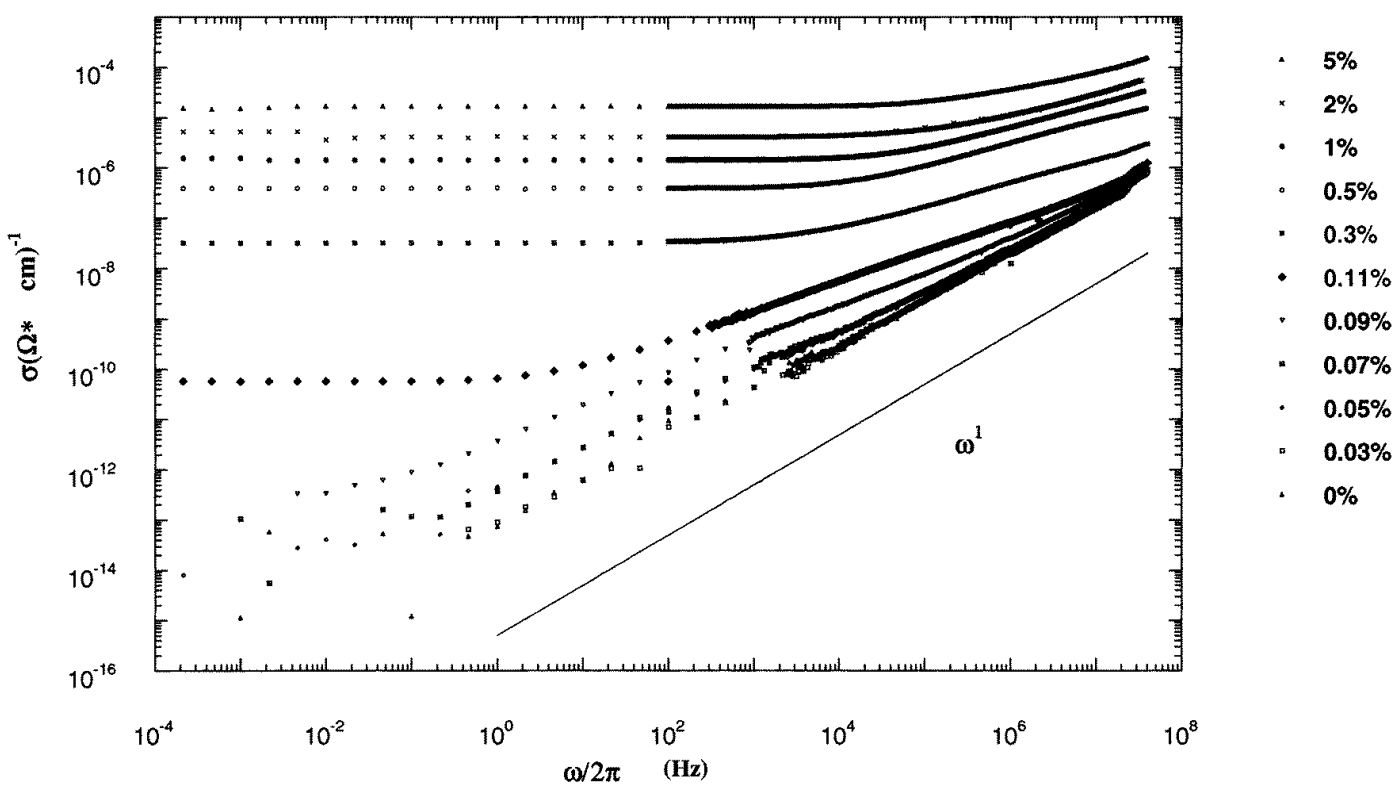

(a)

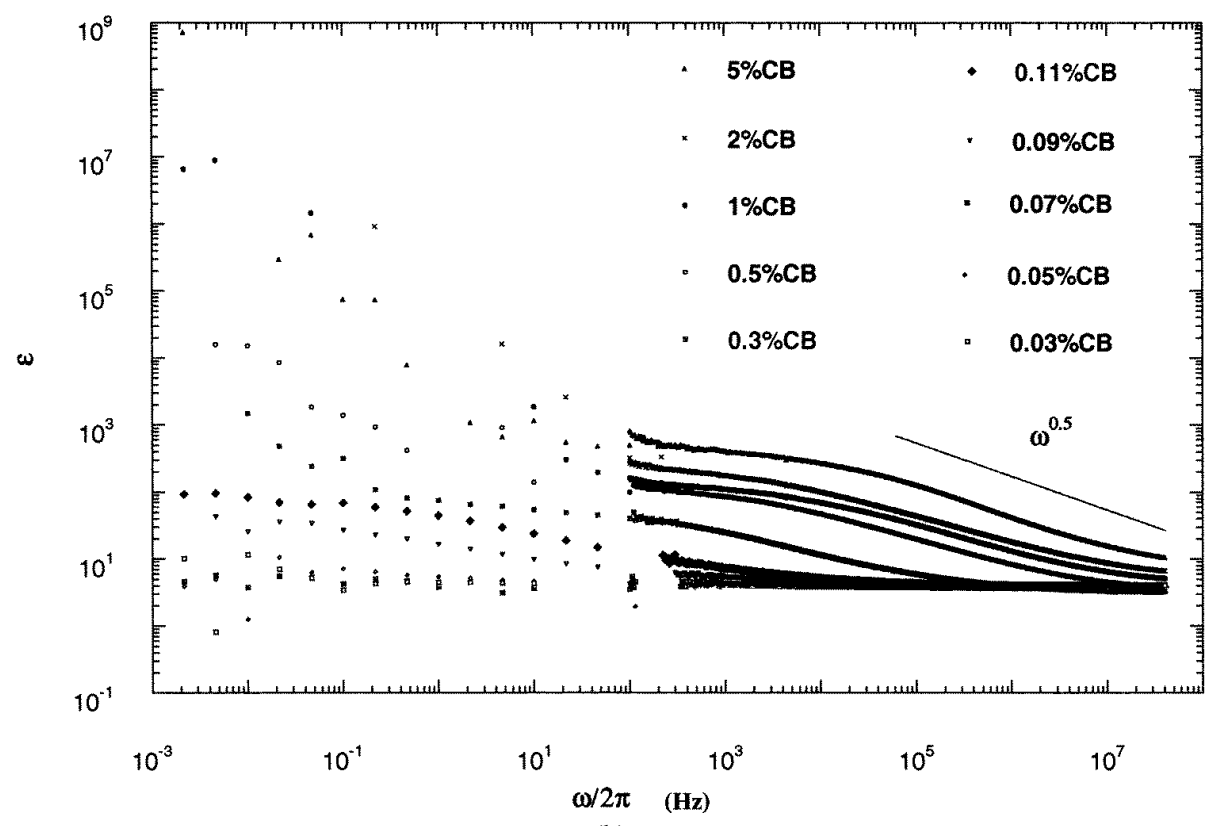

(b)

Figure 5. Angular frequency dependence of (a) ac conductivity, (b) dielectric constant for the 50/50 PS/PMMA blend filled with increasing amounts of BP1000.

that will be designated as regime $\mathrm{C}$. The experimental data of figures 5(a) and 5(b) are fitted by equations 4(a) and (b) in regime A where the dynamical exponent $x$ is slightly higher than 0.5 and $y$ is slightly smaller than 0.5 , indicating that $t \cong s$ (since $x=t / s+t)$ in agreement with the percolation theory for 2D systems [22], and the Bruggeman mean-field theory [31]. The prediction for a 3D system $(x=0.72)$ must obviously be dismissed. This general conclusion is quite consistent with transmission electron microscopy (TEM) images and dc measurements that suggested that most $\mathrm{CB}$ is confined at the interface of the PS/PMMA blends. The exponent $x$ continuously changes from 1 to 0.6 in the intermediate regime $\mathrm{C}(0.11-0.5 \mathrm{wt} \% \mathrm{CB})$. Fluctuations of the dielectric constant data (figure 5(b)) at low frequencies in regime $\mathrm{B}$ will be discussed later.

Below and in the vicinity of $p_{c}$, several transport phenomena may interfere. Far from $p_{c}, \sigma_{e}(\omega)$ is controlled by relaxation processes that exhibit a quasi-universal behaviour $(x \leqslant 1)$. These relaxation phenomena include processes such as the classical trapping and hopping of charges, phonon-assisted quantum mechanical tunnelling through barriers separating the CB clusters and aggregates. Several models have been proposed to account for this behaviour. Since the polymer-CB composites are highly heterogeneous, one cannot consider one relaxation time $(\tau)$ but a wide distribution, that, in some cases, can be represented by a power law distribution

$$
P(\tau) \propto \tau^{-\mu} \quad \text { with } \mu \leqslant 1 .
$$




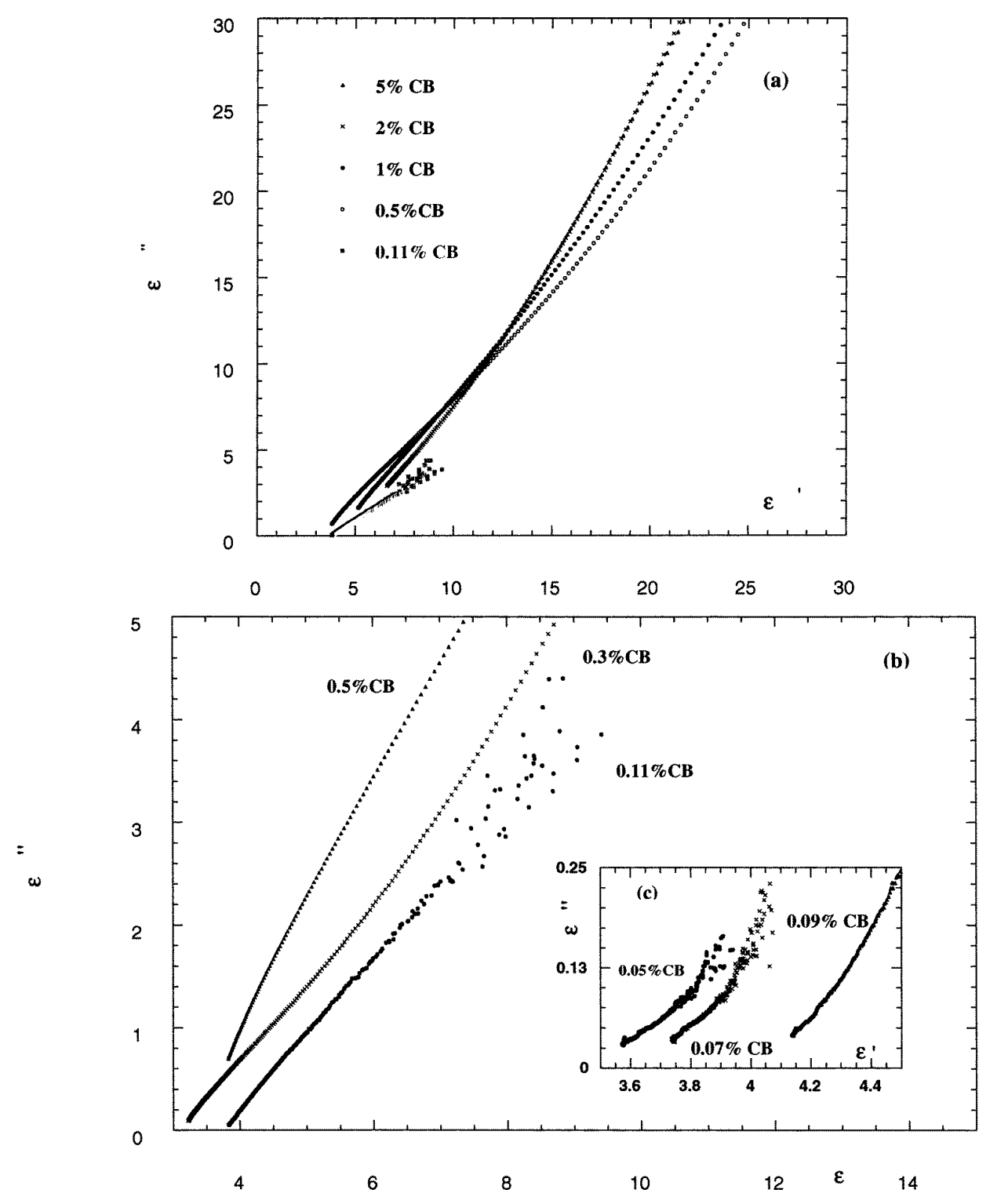

Figure 6. Cole-Cole plots for the 50/50 PS/PMMA blend filled with increasing amounts of BP1000.

A linear frequency dependence of $\sigma(x=1)$ can be observed when the relaxation time is an exponential function of a random variable $\xi$, such that $\tau=\tau_{0} \exp (\xi)$, with $\tau_{0}$ a constant microscopic relaxation time [32]. This situation is typical of the relaxation process in highly heterogeneous materials.

For the lowest CB content $(0-0.05 \mathrm{wt} \% \mathrm{CB})$, the low-frequency conductivity is not reproducible when the experiment is repeated and the samples are changed. A model of conduction via traps proposed by Bouchaud [34] could account for this behaviour. However, not enough data are available with which to consider the reliable statistical analysis of these phenomena. Close to, but below, the percolation threshold (regime $\mathrm{C}$ ), direct tunnelling between adjacent $\mathrm{CB}$ clusters is thought to dominate the conduction process making it difficult to determine the percolation threshold and to change the percolation critical exponents. Sarychev and Brouers [30] have proposed a model to account for this situation. The dielectric exponent $s$ and the dispersion exponent $x$ are different from the usual percolation exponents
(4). Lattice computations yield a value of $x=0.95 \pm 0.05$ [30]. This exponent was found to be close to one for some other polymer-CB composites [36]. In this regime $(C)$, an exponential variation of the dc conductivity typical of tunnelling is observed although the dynamical exponent $x$ is slightly smaller than one.

From the discussion of the three regimes, we can conclude that the percolation threshold lies between $p=$ 0.3 and 0.5 . At $p=0.3$, the conductivity is still dominated by tunnelling (regime C). At $p>0.5$, the data for the conductivity and dielectric constant agree with the percolation model (equation (4), regime A).

Just below and above $p_{c}$, the classical theory predicts a large increase in the dielectric constant (4). Figure 5(b) shows that when the $\mathrm{CB}$ content exceeds $0.11 \mathrm{wt} \%$, large dielectric constants and large fluctuations in this property are observed at frequencies smaller than $1 \mathrm{~Hz}$. Most often, this type of data is not published, being considered as the expression of non-reproducibility due to the limitations of the experimental 


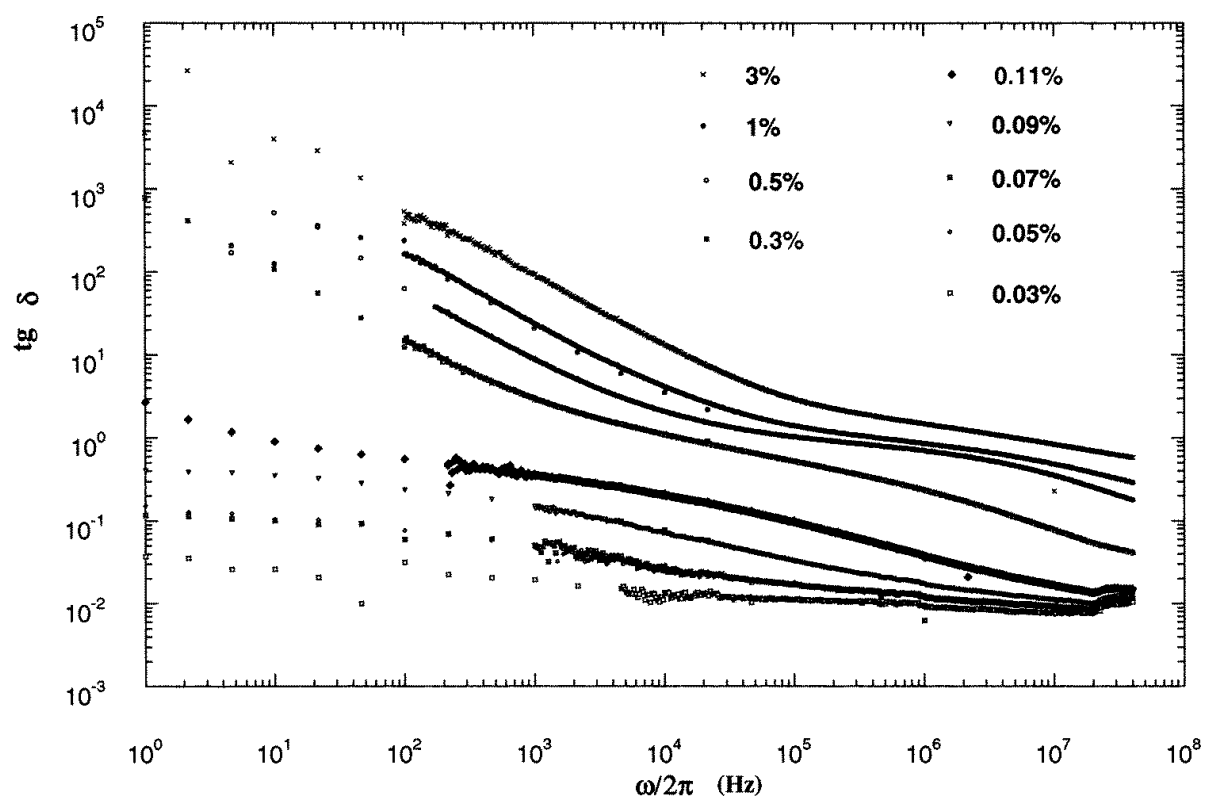

Figure 7. Plot of loss tangent $\delta$ against angular frequency for the 50/50 PS/PMMA blend filled with increasing amounts of BP1000.

techniques and electrode polarization effects. It is worth pointing out that dynamic percolation theory predicts a high fluctuation regime in the case of metal-dielectric composites between the percolation threshold $p_{c}$ and a concentration $p^{*}$, named the 'optical threshold' by Brouers et al [32]. At $p^{*}$ a transition occurs from positive to negative values of the dielectric constant. High negative dc values for the dielectric constant is the signature of metallic conduction. The mean-field theory has been used to discuss the transition at $p^{*}$, at which the mean-field dielectric constant is zero [32]. Between $p_{c}$ and $p^{*}$, large fluctuations are expected between the large positive and large negative values for the dielectric constant. In the critical region close to $p_{c}$, the system is not self-averaging for lengths smaller than the correlation length which tends to very large values. In this region, the oscillating electrical field can probe the metallic or dielectric regions, the permittivity of which is highly negative or highly positive, respectively. Therefore, only some (the positive) values observed in the low-frequency range can be reported in figure 5(b). The missing data are negative values, which cannot be shown on a log-log plot.

In metallic granular films, this transition cannot be observed clearly, since the $\left|p_{c}-p^{*}\right|$ difference depends on the reciprocal resistivity, which is high for metals and much smaller for the polymer-CB composites. Although theoretically predicted, the data reported in this work might be the first experimental evidence for the high-fluctuation regime in the $\left|p_{c}-p^{*}\right|$ range. The probability distribution and the time variations of these fluctuations at a given frequency will be the topic of further investigation.

3.2.3. Cole-Cole and loss angle plots. It is usual practice for insulating, ionic and low conductivity materials to use the Cole-Cole plots to figure the frequency dependence of the complex dielectric constant $\varepsilon^{*}=\varepsilon^{\prime}-j \varepsilon^{\prime \prime}$, where $\varepsilon^{\prime}=\varepsilon$ and $\varepsilon^{\prime \prime}=\sigma / \omega \varepsilon_{0}$. The shape of the Cole-Cole plots in the complex plane has been extensively discussed by Clerc et al [22] for classical percolating systems, that show clearly separated insulating (semiparabolic shape) and conducting regions (hyperbolic shape). At $p=p_{c}$ the plot is linear.

The Cole-Cole plots derived from the experimental conductivity and dielectric constant data (figure 6) confirm that the classical behaviour is not observed, as a result of the coexistence of several conductivity mechanisms in the vicinity of $p_{c}$. Indeed, the Cole-Cole plots in regime $\mathrm{B}$ (figures 6(b) and (c)) have a hyperbolic shape below $p_{c}$, which confirms that the system does not behave as an insulator at this low CB concentration. However, the quasi-straight lines of figures 6(a) and (b), indicate that the geometrical percolation transition occurs in the concentration range of $0.3-0.5 \mathrm{wt} \%$ $\mathrm{CB}$ in agreement with previous conclusion drawn from the ac and dc conductivity data (figures 4 and 5).

Clerc et al [22] have also shown that loss tangent $\delta$, defined as the ratio of the real part and the imaginary part of the complex conductivity, has a universal value $\delta_{c}\left(p_{c}, \omega\right)=$ $(\pi / 2)(1-x)^{-1}$, at $p=p_{c}$. Close to percolation, $\tan \delta \approx 0.8$ (2D) or $\approx 0.25$ (3D). At very low frequencies, $\tan \delta \rightarrow \infty$ for $p>p_{c}$ (the system being purely resistive), and $\tan \delta \rightarrow 0$ for $p<p_{c}$ (the system being purely capacitive). The log-log plot of the tangent of the experimental loss angle $\delta$ is shown in figure 7. For CB concentrations between $0.3-0.5 \mathrm{wt} \%$, a frequency independent dependence over almost two decades is observed for $\tan \delta \approx 0.8$, which confirms the importance of the interface (2D) conductivity.

\section{Conclusion}

The macroscopic electrical properties of polystyrene/poly (methylmethacrylate) blends loaded with special carbon black grade (BP 1000) have been studied. These properties strongly depend on the phase morphology, i.e. the co-continuity of the polymer phases and the selective localization of CB particles at the blend interface. Ternary blends, in which polymer phases and conducting particles 
percolate independently but simultaneously, are the most interesting.

The interfacial localization of $\mathrm{CB}$ particles at the PS/PMMA blend interface dramatically increases the PS/PMMA composition range in which the two polymer phases are continuous. Image analysis of transmission electron micrographs has shown that the interfacial localization of $\mathrm{CB}$ is responsible for a remarkable stabilization of the phase morphology against coalescence during post-annealing treatments. It must be noted that the spontaneous localization of $\mathrm{CB}$ at the interface of binary polyblends is experimentally observed for the first time. In a previous work, dealing with PE/PS blends, BP 1000 was accumulated at the polymer interface as the result of a kinetic control [15] leading to unstable systems which were difficult to reproduce.

The dc measurements have confirmed a very substantial decrease of the CB percolation threshold when localized at the interface of simultaneously continuous PS and PMMA phases.

As result of its composition and morphological characteristics, the system studied in this paper is not a typical percolating system with well-defined concentration threshold and critical exponents. However, for such systems, theoretical considerations have already been published about the concentration range in which the percolation transition occurs and how it determines the mechanism of conduction from ac measurements. This paper reports for the very first time the experimental frequency dependence of the conductivity and the dielectric constant from $p \ll p_{c}$ to $p>p_{c}$ over more than 10 decades $\left(10^{-4}-10^{8} \mathrm{~Hz}\right)$. From the detailed analysis of these measurements, three regimes of electrical conduction have been identified for 50/50 PS/PMMA blends loaded with CB (0-5 wt\%):

(1) In the range of $0.5-5 \mathrm{wt} \% \mathrm{CB}$, well above the percolation threshold, the conduction mechanism is clearly in agreement with the classical percolation theory for $2 \mathrm{D}$ systems.

(2) In the $0.11-0.5 \mathrm{wt} \% \mathrm{CB}$ range, in the vicinity of the percolation threshold, direct tunnelling between adjacent clusters dominates the conduction process. This behaviour makes the determination of $p_{c}$ difficult and modifies the percolation critical exponents.

(3) For CB contents smaller than 0.09 wt $\%$, well below the percolation threshold, the low-frequency trapping and hopping conductivity is no longer reproducible, being dependent on the experiment and the sample. Such a non-self-averaging physical situation is now the subject of a number of theoretical and experimental investigations [35].

The detailed analysis of the ac data interestingly shows that the 50/50 PS/PMMA blends loaded with small amounts of $\mathrm{CB}$, i.e. $0.11-0.3 \mathrm{wt} \%$, exhibit stable and reproducible electrical and dielectric properties over nearly eight decades.

\section{Acknowledgments}

The authors are very much indebted to the 'Services Fédéraux des Affaires Scientifiques, Techniques et Culturelles' for support in the frame of the 'Pôles d'Attraction Interuniversitaires: PAI 4/11'. They warmly thank R Pirard from the 'Service de Génie Chimique' (University of Liège Belgium) for dielectric measurements.

\section{References}

[1] Bekele S and Lee V W 1986 US Patent 4592861

[2] Wessling B 1986 European Patent 181587

[3] Felter R E, Markey D A and Musser D L 1990 US Patent 4976890

[4] Moddelmog K, Niedermanner H and Zimmer G 1989 Ger. Patent DD 272772

[5] Karger-Kocsis J, Kozma B, Schober M, Kasza K and Nadas D 1989 Hung. Patent 47615

[6] Scmidchten H M and Hammersley J 1956 Proc. Camb. Phil. Soc. $\mathbf{5 3} 629$

[7] Ponomarenko A T, Shevchenko V G and Enikolopyan N S 1990 Adv. Polym. Sci. 96125

[8] Miyasaka K, Watanabe K, Jojima E, Aida H, Sumita M and Ischikawa K 1982 J. Mat. Sci. 171610

[9] Sumita M, Abe H, Kayaki H and Miyasaka K 1986 J. Macromol. Sci.-Phys. B 25171

[10] Sicar A and Lamond T G 1978 Rubb. Chem. Technol. 51126

[11] Verhelst W F, Wolthuis K G, Voet A, Ehrburger P and Donnet J B 1977 Rubb. Chem. Technol. 50735

[12] Gubbels F, Jérôme R, Teyssié R, Vanlathem E, Deltour R, Calderone A, Parenté V and Brédas J L 1994 Macromolecules 271972

[13] Gubbels F, Blacher S, Vanlathem E, Jérôme R, Brouers F, Deltour R and Teyssié Ph 1995 Macromolecules 281559

[14] Soares B G, Gubbels F, Jérôme R, Teyssié Ph, Vanlathem E and Deltour R 1995 Polym. Bull. 35223

[15] Gubbels F, Jérôme R, Vanlathem E, Deltour R, Blacher S and Brouers F 1998 Chem. Mat. 101227

[16] Balberg I 1987 Phys. Rev. Lett. 591305

[17] Kawamoto H 1982 Carbon Black-Polymer Composites ed E K Sichel (New York: Dekker)

[18] Carmona F, Barreau F, Delhaes P and Canet R 1980 Phys. Lett. 41 L-534

[19] Balberg I and Bozowski S 1982 Solid State Commun. 44551

[20] Chung K T, Sabo A and Pica A P 1982 J. Appl. Phys. 53 6867

[21] Stauffer and Aharony A 1991 Introduction to Percolation Theory (Philadelphi, PA: Taylor and Francis) and references therein

[22] Clerc J P, Giraud G, Laugier J M and Luck J M 1990 Adv. Phys. 3919

[23] Abeles B, Sheng P, Coutts M D and Arie Y 1975 Adv. Phys. 24407

[24] Sichel E K and Gittelman J I 1982 J. Electron. Mater. 11699

[25] Sheng P and Klafter J 1983 Phys. Rev. B 272583

[26] Hakizabera P 1993 Thèse de Doctorat Université Libre de Bruxelles

Hakizabera P, Deltour R and Brouers F 1995 Mat. Res. Soc. Symp. Proc. $\mathbf{3 6 7} 425$

[27] Feng S, Halperin B I and Sen P N 1987 Phys. Rev. B 35197

[28] Sen P N, Roberts J N and Halperin B I 1987 Phys. Rev. B 32 3306

[29] Kogut P M and Straley J P 1979 J. Phys. C: Solid State Phys. 122151

[30] Sarychev A K and Brouers F 1994 Phys. Rev. Lett. 732895

[31] Brouers F 1986 J. Phys. C: Solid State Phys. 12783

[32] Brouers F, Clerc J P and Giraud G 1991 Phys. Rev. B 445299

[33] Brouers F, Royen I, Blacher S, Pirard R, Gubbels F, Jérome R and Sarychev A 1996 Mat. Res. Soc. Symp. Proc. 411329

[34] Bouchaud J P 1995 Levy Flights and Related Topics in Physics ed M Slesinger, G Zaslavsky and U Frisch (Berlin: Spinger) p 239

[35] Bardou F 1997 Europhys. Lett. 39239 